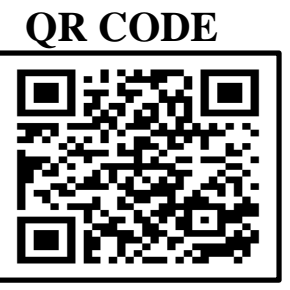

\title{
The Toxicity of Corticosteroids
}

\section{DAVID SMITH' ${ }^{1}$ VENU SEN ${ }^{2}$}

Minimization of steroid therapy has always been one of the main objectives of immunosuppressive protocols after kidney transplantation, due to numerous side effects. The use of a further reduced daily dose of steroids is considered by many to be a fair compromise between toxicity and efficacy. Unfortunately, the great inter-individual variability of the pharmacokinetics of steroids does not prevent the appearance of major side effects in a variable percentage of patients, even with the low dose used. A drug interaction between steroids and drugs used after transplantation can also contribute to increasing daily exposure to the drug. Steroid discontinuation is still the only procedure capable of achieving the desired goal. This procedure is associated with a greater risk of acute rejection, without however reducing the survival of the transplant. It should be offered to patients at low immunological risk. Early discontinuation, during the first week of transplantation, is also the modality suggested by some guidelines, although a later suspension also offers excellent results. Induction therapy is always recommended in the case of early discontinuation.

KEYWORDS: Corticosteroids, Steroids, Drug Toxicity

\section{INTRODUCTION}

Synthetic glucocorticoids were introduced in the late 1950 and are the drugs used for the longest time after kidney transplantation. At first, prednisone, or its equivalent, was administered once or twice a day, at a dose of $15 \mathrm{mg} .{ }^{1}$ The first studies immediately documented two important aspects related to their clinical use: The first was that steroids were effective in preventing acute rejection only when used in combination with other drugs, and not when used alone. The second one concerned the appearance of serious side effects when used for a longer period of time. The latter problem was so important that it became the subject of many studies, and soon new therapeutic schemes were proposed with the aim of containing this problem that reduced the quality of life of patients. ${ }^{2}$ It was reported in a study that there is no consensus on what the minimum required dose of steroid should be, the minimum dose required may differ from subject to subject and gradual steroid reduction leads to an increased risk of acute rejection. ${ }^{3}$

The appearance of cyclosporine gave rise to a new line of clinical research based on the possibility of implementing immunosuppressive protocols without steroids. ${ }^{4}$ This was immediately received with great interest by clinicians because it allowed to eliminate, or reduce, the known side effects related to the prolonged use of the steroid. ${ }^{5}$ There is no doubt that steroid-free protocols increase the risk of acute rejection. ${ }^{6}$ Unfortunately, they do not even reduce patient mortality, as expected, given the significant reduction of some of the main risk factors for cardiovascular disease, always reported in these cases. ${ }^{7}$ On the basis of this evidence, steroid-free protocols today must have, as the only main objective of avoiding the known side effects, in order to improve the patient's quality of life. At this point, the problem arises of what is the real incidence and severity of these side effects today. Today, compared to past decades, the daily dosage of prednisone or its equivalent has gradually decreased. Therefore, it is questionable whether such a low dose is in any case toxic for the patient or is free from important side effects.

A study showed that $5 \mathrm{mg}$ of steroid per day, compared to discontinuation, did not increase the risk of diabetes mellitus. ${ }^{8}$ This conclusion confirmed a previous study that evaluated the effect of different doses of steroid on peripheral insulin resistance, also in renal transplant patients. The study showed that insulin sensitivity improved with the gradual reduction of doses. ${ }^{9}$ However, these results were contradicted by another study, published in 2016. ${ }^{10}$ Even low doses of steroid can affect the onset of diabetes, especially if taken for a long period of time, and if taken in combination with other pro-diabetic drugs. ${ }^{.1}$ Another side effect that could benefit from the discontinuation of the steroid concerns bone

(C) David Smith et al. This is an open access article distributed under the terms of the Creative Commons Attribution License CC-BY-NC 4.o, which permits unrestricted use, distribution and reproduction in any medium, provided the use is not commercial and the original author(s) and source are cited. Submitted on: 29-Nov-2021; Accepted on: 26-Jan-2022 
pathology, in particular osteoporosis. As is known, this pathology carries a high risk of spontaneous vertebral or peripheral fractures, with serious repercussions on the patient's quality of life. It is agreed that the greatest loss of bone density occurs during the first 6-12 months of transplantation, a period in which steroid doses are usually higher. ${ }^{12}$ It has been calculated that avoiding the steroid can significantly reduce the risk of osteoporosis, but not eliminate it completely, given the pathogenetic role played by other factors. ${ }^{13}$ In one study, it was documented that a daily dose between 2.5-7.5 mg corresponded to an increased risk of $18 \%$ for peripheral fractures, and $54 \%$ for vertebral fractures. ${ }^{14}$

Thus, using low doses of the steroid reduces the risk of osteoporosis, but it cannot be argued that it is equivalent to not using it at all. On the contrary, the increase in risk remains significant even at low doses and will probably be even greater in the categories of more exposed subjects. Another finding that emerged from this study is that the risk of steroid-induced fractures does not increase during the course of therapy, and rapidly decreases after its discontinuation..$^{15}$ Another aspect to focus on is offered to us by daily clinical practice. It is common experience that the same steroid dose, even if low, can have very different side effects from patient to patient. This evidence should suggest the need to tailor the steroid dose.

In the field of transplantation, the two most commonly used synthetic glucocorticoids are prednisone and prednisolone. The latter is the main corticosteroid present in plasma and is the only one capable of crossing the cell membrane and producing the expected pharmacological effects. Prednisone and prednisolone both have rapid intestinal absorption both have a high bioavailability. After transplantation, the elimination of prednisolone is slowed down by the concomitant use of several drugs. The elimination of the drug is not constant, it decreases after the first year. ${ }^{16}$ These pharmacokinetic notes document the complexity of steroid metabolism which corresponds to a wide inter-individual variability, and consequently a different toxicity from subject to subject. Many studies, especially in the past, have evaluated whether there was a correlation between the degree of exposure to prednisolone and the occurrence of one or more side effects. In this context, one of the most investigated complications was Cushing's Syndrome. It has been hypothesized that the appearance of these manifestations could depend on greater exposure to the drug, despite equally low daily doses. In some patients, smaller doses are sufficient to obtain the desired pharmacological effect. In fact, some pharmacokinetic studies have confirmed a correlation between high exposure to prednisolone, and the appearance of Cushing-like side effects. ${ }^{17}$ These findings, of certain clinical relevance, were confirmed $^{18-20}$, but also denied by some studies ${ }^{21-23}$, and therefore the problem remains open to different interpretations. Greater steroid exposure was also reported in subjects who developed diabetes after transplantation. ${ }^{24}$ Pharmacodynamic studies carried out on cultures of peripheral lymphocytes have also found a very different sensitivity to the action of the steroid from subject to subject. Consequently, different doses of the drug should be required to achieve the same pharmacological effect. ${ }^{25}$ All these aspects show us the complexity of the problem, and how the empirical reduction of the dose of prednisolone alone cannot guarantee the same efficacy and safety for all patients.

All clinical studies that have dealt with the discontinuation of the steroid have always excluded patients at high immunological risk. However, a recent meta-analysis reported results in favor of steroid discontinuation even in subjects considered to be at high immunological risk. ${ }^{26} \mathrm{GN}-\mathrm{IgA}$ is also considered as a contraindication to the steroid discontinuation. An association was reported between steroid discontinuation and an increased risk of GN-IgA recurrence. ${ }^{27}$ Subsequently, many other studies have confirmed this finding ${ }^{28-30}$, while a few others have excluded it. ${ }^{31}$ The ABO-incompatible living transplantation represents one of the contraindications to steroid-free therapy as it is considered to be at greater risk of acute rejection. Today we know that late discontinuation has an undoubted advantage, that of selecting patients to discontinue the steroid, and this results in a lower incidence of acute rejection compared to early discontinuation..$^{32}$ The selection consists in excluding from discontinuation the patients with one or more episodes of acute rejection, or those impaired or unstable renal function. In some protocols, a followup renal biopsy is also provided before discontinuation of the steroid. ${ }^{33}$

In clinical practice, the late discontinuation of the steroid results in greater difficulty in outpatient management of the patient, precisely in a period in which simplification of monitoring procedures should be applied instead. Early discontinuation allows to avoid many of the limitations reported above and, 
therefore, simplifies the clinical management of the patient, in the context of, as already mentioned, a greater risk of acute rejection. However, in early discontinuation episodes, acute rejection occurs mainly during the first month, when outpatient checks are frequent and, therefore, without repercussions on normal procedures. Today, early discontinuation is preferred to immediate discontinuation because it facilitates the clinical management in the immediate post-transplantation period and reduces the risk of acute rejection. ${ }^{34}$ The latter point was further confirmed by a recent randomized study comparing the two different ways of avoiding steroids, with the primary objective being the incidence of diabetes, which was comparable. ${ }^{35}$

A further aspect to be considered concerns the necessity or not of induction therapy in candidates for steroid discontinuation after transplantation. Induction therapy is strongly recommended by current guidelines in cases of early steroid discontinuation ${ }^{36}$ and has always been included in all proposed protocols over the years. In cases of late discontinuation, this recommendation does not apply, although it may be useful in increasing the percentage of subjects eligible for discontinuation, due to a likely lower incidence of acute rejection and better renal function.

\section{CONCLUSION}

The results obtained do not allow to reach a single and definitive conclusion, due to the many aspects involved, and to which more or less importance can be given. In summary, however, it can be stated that steroid toxicity is a complex effect that is difficult to measure with a single parameter. High interindividual variability of prednisolone pharmacodynamics should suggest personalization of therapy. The reduction of the daily dose of prednisone or its equivalent has certainly reduced the degree of toxicity of the drug, but it has not eliminated the problem of side effects and their impact on the quality of life of many patients and on their cardiovascular risk profile. The best solution to the problem could be offered by the discontinuation of the drug, which is possible in many patients classified as patients at low immunological risk. In these patients, the increased risk of acute rejection may be largely offset by the benefits deriving from the absence of steroid toxicity.

\section{REFERENCES}

1. Murray JE, Merrill JP, Harrison H et al. Prolonged Survival of Human-Kidney Homograft by Immunosuppressive Drug Therapy. N Engl J Med. 1963; 268: 1315-23.

2. Siegel RR, Luke RG, Hellebusch AA. Reduction of Toxicity of Corticosteroid Therapy after Renal Transplantation. Am J Med. 1972;53:159-69.

3. Turcotte JG, Dickerman RM, Harper ML. Minimum steroid requirements in the late post-transplant period. Transplant Proc. 1975;7(1):83-8.

4. Calne RY, Thiru S, McMaster P et al. Cyclosporin A in Patients receiving Renal allograft from Cadaver Donors. The Lancet 1978;312(8104):1323-7.

5. Rhen T, Cidlowski JA. Antiinflammatory action of Glucocorticoids- New Mechanisms for Old Drugs. N Engl J Med. 2005; 353:1711-23.

6. Haller MC, Royuela A, Nagler EV et al. Steroid avoidance or withdrawal for kidney transplant recipients. Cochrane Database Syst Rev. 2016;(8):CDoo5632.

https://doi.org/10.1002/14651858.CDoo5632.pub3

7. Knight SR, Morris PJ. Steroid Avoidance or Withdrawal after renal transplantation Increases the Risk of Acute Rejection but Decreases Cardiovascular Risk. A Meta-Analysis. Transplantation 2010;89: 1-14.

8. Pirsch JD, Henning AK, First MR et al. New-Onset Diabetes After Transplantation: Results From a Double-Blind Early Corticosteroid Withdrawal Trial. Am J Transplant. 2015; 15: 1982-90. https://doi.org/10.1002/10.1111/ajt.13247

9. Midtvedt K, Hjelmesaeth J, Hartmann A. Insulin Resistance after Renal Transplantation: The Effect of Steroid Dose Reduction and Withdrawal. J Am Soc Nephrol. 2004; 15:3233-9.

10. Thomusch O, Wiesener M, Opgenoorth $\mathrm{M}$ et al. Rabbit ATG or Basiliximab Induction for Rapid Steroid Withdrawal after Renal Transplantation (Harmony): an open-label, multicentre, randomised controlled trial. Lancet 2016; 388:3006-16. https://doi.org/10.1016/So140-6736(16)32187-o 11. van Raalte DH, Brands M, van der Zijl NJ, Muskiet $\mathrm{MH}$, Pouwels PJ et al. Low-dose glucocorticoid treatment affects multiple aspects of intermediary metabolism in healthy humans: a randomised controlled trial. Diabetologia 2011;54:2103-12.

12. Taweesedt PT, Disthabanchong S. Mineral and Bone Disorder after Kidney Transplantation. World J Transplant. https://doi.org/10.5500/wjt.v5.i4.231.

$2015 ; 5(4): 231-42$. 
13. Nikkela LE, Mohanb S, Zhang A et al. Reduced Fracture Risk With Early Corticosteroid Withdrawal After Kidney Transplant. Am J Transplant. 2012;12: 649-59.

14. van Staa TP, Leufkens HGM, Abenhaim L et. Oral corticosteroids and fractures risk: relationship to daily and cumulative doses. Rheumatology 2000;39:1383-9.

15. van Staa TP, Leufkens HGM, Abenhaim L et. Use of oral corticosteroids and risk of fractures. J Bone Miner Res. 2000; 15:993-1000.

16. Bergmann TK, Barraclough KA, Lee KJ, Staatz CE. Clinical Pharmacokinetics and Pharmacodynamics of Prednisolone and Prednisone in Solid Organ Transplantation. Clin Pharmacokinet. 2012; 51:711-41.

17. Bergrem $\mathrm{H}$, Jervell JAK, and Flatmark A. Prednisolone pharmacokinetics in cushingoid and non-cushingoid kidney transplant patients. Kidney International 1985;27:459-64.

18. Bergmann TK, Isbel N, Ostini R et al. Exploratory Study of Total and Free Prednisolone Plasma Exposure and Cushingoid Appearance, Quality of Life and Biochemical Toxicity in Adult Male Kidney Transplant Recipients. Clin Drug Investig. 2015;35:743-50.

19. Potter JM, McWhinney BC, Sampson L, Hickman $\mathrm{PE}$. Area-under-the-curve monitoring of prednisolone for dose optimization in a stable renal transplant population. Ther Drug Monit. 2004;26:408-14.

20. Kozower M, Veatch L, Kaplan MM. Decreased clearance of prednisolone, a factor in the development of corticosteroid side effects. J Clin Endocrinol Metab. 1974;38:407-12.

21. Frey FJ, Amend WJ, Lozada F, et al. Pharmacokinetics of prednisolone and endogenous hydrocortisone levels in cushingoid and noncushingoid patients. Eur J Clin Pharmacol. 1981;21(3):235-42.

22. Benet LZ, Frey FJ, Amend WJ Jr, et al. Endogenous and exogenous glucocorticoids in cushingoid patients. Drug Intell Clin Pharm. 1982;16(11):863-8.

23. Ost L, Bjorkhem I, von Bahr C. Clinical value of assessing prednisolone pharmacokinetics before and after renal transplantation. Eur J Clin Pharmacol. 1984;26(3):363-9.

24. Ionita IA, Ogasawara $K$, Gohh RY et al. Pharmacokinetics of Total and Unbound Prednisone and Prednisolone in Stable Kidney Transplant Recipients with Diabetes Mellitus. Ther Drug Monit. 2014; 36: 448-55. https://doi.org/10.1097/FTD.oooooooooooooo45

25. Muhetaer G, Takeuchi H, Unezaki S et al. Clinical Significance of Peripheral Blood Lymphocyte Sensitivity to Glucocorticoids for the Differentiation of
High-risk Patients With Decreased Allograft Function After Glucocorticoid Withdrawal in Renal Transplantation. Clin Ther. 2014; 36:1264-72. https://doi.org/10.3727/215517912X639423

26. Song TR, Jiang YM, Liu JP et al. Steroid withdrawal or avoidance is safe in high-risk kidney transplants: A systematic review and meta-analysis. Kaohsiung J Med Sci. 2019; 35 :350-7. https://doi.org/10.1002/kjm2.12064 27. Clayton P, McDonald S, Chadban S, Steroids and Recurrent IgA Nephropathy After Kidney Transplantation. Am J Transplant 2011;11: 1645-9.

28. Di Vico MC, Messina M, Fop F et al Recurrent IgA nephropathy after renal transplantation and steroid withdrawal. Clin Transplant 2018;32: e13207.

29. Leeaphorn N, Garg N, Khankin VE et al Recurrence of IgA nephropathy after kidney transplantation in steroid continuation versus early steroid-withdrawal regimens: a retrospective analysis of the UNOS/OPTN database. Transplant International 2018; 31:175-86. https://doi.org/10.1111/tri.13075

30. Von Visger JR, Gunay Y, Andreoni KA et al The risk of recurrent IgA nephropathy in asteroid-free protocol and other modifying immunosuppression. Clin Transplant. 2014: 28: 845-54.

31. Barbour S, Djurdjev O, Gill J.S. et al. A propensity score matched analysis shows no adverse effect of early steroid withdrawal in non-diabetic kidney transplant recipients with and without glomerulonephritis. Kidney International 2019;96:460-9. https://doi.org/10.1016/j.kint.2019.02.041

32. Vincenti F, Schena FP, Paraskevas S et al. A randomized, multicenter study of steroid avoidance, early steroid withdrawal or standard steroid therapy in kidney transplant recipients. Am J Transplant. 2008; 8:307-16.

33. Wehmeier C, Hirt-Minkowski P, Amico P et al. Successful steroid withdrawal guided by surveillance biopsies-A single-center experience. Clin Transplant. 2018;32(3):e13181. https://doi.org/10.1111/ctr.13181

34. Vincenti F, Schena FP, Paraskevas S, Hauser IA, Walker RG etal. A Randomized Multicenter Study of Steroid Avoidance, Early Steroid Withdrawal or Standard Steroid Therapy in Kidney Transplant Recipients. Am J Transplant. 2008;8:307-16.

35. Mourad G, Glyda M, Albano L, et al. Incidence of Posttransplantation Diabetes Mellitus in De Novo Kidney Transplant Recipients Receiving ProlongedRelease Tacrolimus-Based Immunosuppression With 2 Different Corticosteroid Minimization Strategies: ADVANCE, A Randomized Controlled Trial. Transplantation 2017; 101:1924-34. https://doi.org/10.1097/TP.oooooooooooo1453 
36. KDIGO Clinical Practice Guideline for the Care of Kidney Transplant Recipients. Am J Transplant. 2009; 9(suppl 3):S10-S13. 